MODELING, IDENTIFICATION AND CONTROL, 1996, VOL. 17, NO. 4, 261-278

doi:10.4173/mic.1996.4.2

\title{
Process Identification in On-Line Optimizing Control, an Application to a Heat Pump
}

\author{
MORTEN CHRISTIAN SVENSSON†
}

Keywords: System Identification; dynamic optimization; heat pump

The objective of this paper is to focus on on-line state and parameter estimation in connection with on-line model-based optimizing control of continuous processes. A nonlinear programming approach is used to estimate unmeasured state variables and parameters in systems modelled by nonlinear differential-algebraic equations. The nonlinear dynamic model is discretized by orthogonal collocation on finite elements, and the moving-horizon approach is used to reduce the dimension of the final optimization problem.

A priori parameter information is included in the minimization criterion (Bayes estimation), and this makes the estimation problem more robust with respect to missing process excitations and over-parameterization. The a priori parameter covariance matrix is treated as a tuning matrix, where the diagonal elements can be set acording to the amount of information in the measurements.

The updated steady-state part of the process model is used to optimize the economic performance of the process, where new optimum set-points are calculated for the regulatory control system. The sequential quadratic programming method (SQP) is used to solve this nonlinear optimization problem, where the objective function, the model equations, and the operational feasibility constraints are solved simultaneously in an "infeasible path" approach.

The identification and on-line optimizing control approach are illustrated with an example from an experimental water-to-water heat pump unit.

\section{Introduction}

Increased energy and raw material costs have afforded a strong incentive to engineers to develop well-posed control schemes which give greater economic return in process operation. By continuously maintaining the plant at its optimum operating condition, despite changing environmental conditions and equipment behaviour, it is possible to achieve an important performance improvement. Continuous tracking and driving the process to its best operating conditions when such changes occur are termed on-line optimizing control. Design and implementation of on-line optimizing control are strongly affected by the characteristics of the disturbances mentioned above. Steady-state optimizing control, which will be focused on in this paper, is well-justified if the disturbances are both relatively slow (compared to the process dynamics) and have a significant impact on the optimum economic performance of the plant.

As reviewed by Arkun and Stephanopoulos (1980), various optimizing control methods have been proposed. Existing methods fall into two categories which differ in modelling complexity and implementational strategies. In the direct search methods (Edler et al. 1970, Box and Draper 1968) the on-line optimization is done directly on the process without explicitly using any process model. The changes in the manipulated

Received 25 September 1995.

$\dagger$ †Department of Electrical Engineering, School of Engineering and Food Science, Høgskolen i Sør-Trondelag, Trondheim, Norway. 
variables or set-points are made at intervals sufficiently far apart to allow the process to reach steady-state. The objective function is measured and its sensitivity is used to readjust the manipulated variables or set-points. Direct search methods are easy to implement and are suitable for processes with few degrees of freedom and rapid dynamics. If disturbances arrive at a rate preventing the plant from reaching a steady-state, then these methods are not applicable.

The indirect methods use an explicit process model, and the optimum operating conditions for the process are determined based on this mathematical model and an appropriate performance criterion. A rigorous model will generally represent the plant better than a simple one. However, when it comes to on-line optimization, a rigorous model may have the disadvantage of requiring significantly longer computation time. On the other hand, if the employed model is too simple, it may not provide an accurate enough representation of the plant behaviour and its optimization may result in non-optimum, or in some cases physically infeasible operating conditions being calculated. Several optimization techniques can be used to solve the nonlinear optimization problem (Edgar et al. 1989). However, the sequential quadratic programming method, which is used in this paper, seems promising in terms of computational efficiency and robustness. In this method the performance criterion, the steady-state model equations, and the operational feasibility constraints are solved simultaneously in an "infeasible path" approach.

Mismatch between model and plant can be due to a number of factors such as: uncertain parameters, unknown state variables, unmeasured disturbances, error in the model structure, and measurement noise. Proper adaptation schemes, where the model parameters are udpated on the basis of recent measurements, need to be incorporated into the model-based optimization control approach to minimize the plant/model mismatch. There exist several approaches to cope with this problem, where all are adaptive in nature but differ in their adaptation schemes. One approach is steady-state identification, where the parameters and unmeasured states in a steady-state model are estimated at steady-state conditions. Different parameter estimation techniques such as least square, maximum likelihood, etc, can be used to update the process model. Kim et al. (1990) compared simultaneous and sequential data reconciliation and parameter estimation for steady-state systems using nonlinear programming techniques. MacDonald et al. (1988) did the same comparison but they used the algorithm proposed by Britt and Leucke (1973). The steady-state identification method is subjected to steady-state operating conditions, which necessitates a criterion for determination of steady-state (Narasimhan et al. 1986, Narasimhan and Mah 1987). Two major drawbacks of these steady-state identification methods are that they might be slow if one waits for the plant to settle to steady-state after each change, and if the plant is subject to persistent disturbances which prevent it from reaching any steady-state, they will not be sufficient.

Dynamic identification methods do not suffer from these drawbacks. Measurements are used during transients and not only at steady-state to estimate state variables and parameters. This makes the convergence to the optimum operating region faster than the steady-state identification methods. An adaptive method is developed by Bamberger and Isermann (1978) for on-line steady-state optimization of slow dynamic processes. They identify the parameters of a discrete dynamic second-order Hammerstein model of the process. The Hammerstein representation is linear in terms of the unknown parameters of the system and on-line identification techniques such as recursive least squares, approximate maximum likelihood, and instrumental variables apply. The steady-state part of the model is used in the steady-state optimization to calculate new 
optimum values for the manipulated variables. Jang et al. (1987) use a similar two-phase approach to control chemical processes at their optimum operating conditions. The first phase consists of on-line state and parameter identification of an approximate nonlinear dynamic process model. In the second phase, the optimum operating strategy is determined by integrating and optimizing this identified process model over a selected time horizon into the future. They use a moving-horizon identification method as an alternative to the recursive identification method. The moving-horizon identification approach has much in common with off-line identification, where the measurements are treated like a batch for a given time horizon. In on-line identification this time horizon is moving such that it always contains the most recent measurements. Benefits with a moving-horizon identification method compared to recursive methods are:

- The method can handle nonlinear fundamental physical models.

- State variable and parameter constraints can easily be incorporated in the identification problem.

- The identification problem can be reconfigurated on-line with respect to both criterion and process model.

Jang et al. (1986) compare this nonlinear state and parameter estimation to the extended Kalman filter, and they conclude that using a nonlinear programming approach is superior in terms of response to changes in parameters and robustness in the presence of modelling errors and strong nonlinearities.

Kim et al. (1991) investigate a sequential approach for data reconciliation and parameter estimation, where the numerical integration of a nonlinear dynamic process model is nested within a nonlinear programming algorithm. Liebman et al. (1992) present a simultaneous approach for solving the general nonlinear dynamic data reconciliation and parameter estimation problem in an on-line environment. They use an "infeasible path" algorithm, where the model is discretized to form algebraic equations which are imposed as equality constraints in a nonlinear programming formulation. The discretization is accomplished using orthogonal collocation on finite elements. Similar identification schemes are used in nonlinear model-predictive control (Sistu et al. 1991, Eaton et al. 1990, Biegler et al. 1991).

Identification is needed only when there is a substantial difference between the output of the model and that of the process. Sistu et al. (1990) use a statistical analysis-based approach to activate the identification scheme. Without a sufficient amount of persistent excitations of the process so that enough process modes are excited, the parameters of a dynamic model may become unidentifiable. If the process is not naturally persistently excited by disturbances or manipulated variables, this problem of near or true unidentifiability results in a model which no longer reflects the process accurately (Jang et al. 1987).

This paper follows the approach of decomposing the identification and optimization phase of optimizing control. To achieve faster convergence to the optimum operating region, and to cope with persistent disturbances, a nonlinear non-steady-state process model is identified in the identification phase. The updated steady-state part of the model is then used in the optimization phase, where the economic performance of the process is optimized. Both the identification and the optimization problem are solved within an "infeasible path" nonlinear programming approach. A priori parameter information is included in the identification criterion in the identification phase, which makes the estimation problem more robust with respect to missing process excitations and 
over-parameterization. The proposed approach is illustrated with an example from an experimental water-to-water heat pump unit.

\section{State and parameter estimation formulation}

The objective of the model identification phase is to minimize the plant/model mismatch by using state variables and model parameters as decision variables. The general nonlinear dynamic identification problem can be written as:

subject to

$$
\min _{\hat{\boldsymbol{\theta} . \hat{x}}} \boldsymbol{\Phi}\left(\hat{\boldsymbol{\theta}}, \hat{\boldsymbol{x}}_{0}, \hat{\boldsymbol{y}}_{1}, \ldots, \hat{\boldsymbol{y}}_{N}\right)
$$

$$
\begin{aligned}
& \frac{d \hat{x}}{d t}-f[\hat{x}(t), \hat{u}(t), \hat{v}(t), \hat{\theta}]=0 \\
& \hat{y}(t)-z[\hat{x}(t), \hat{u}(t), \hat{v}(t), \hat{\theta}]=0 \\
& h[\hat{x}(t), \hat{u}(t), \hat{v}(t), \hat{\theta}]=0 \\
& g[\hat{x}(t), \hat{u}(t), \hat{v}(t), \hat{\theta}] \leq 0
\end{aligned}
$$

where $\hat{\boldsymbol{\theta}}$ is an estimate of the true parameters, $\hat{\boldsymbol{x}}$ is an estimate of the true states, $\hat{\boldsymbol{y}}_{\boldsymbol{i}}=\hat{\boldsymbol{y}}\left(\boldsymbol{t}_{i}\right)$ is an estimate of the true measurements at time $t_{i}, \hat{x}_{0}$ is an estimate of the true unmeasured initial conditions of the state variables, $\hat{\boldsymbol{u}}$ is an estimate of the true manipulated variables, and $\hat{v}$ is an estimate of the true disturbances.

The above problem formulation represents a nonlinear programming approach to model identification, and the identification criterion $\Phi$ is not required to take any special form. In a model based on "first principles" available a priori information is included in the model in a natural manner. A priori parameter estimates and a priori estimates of the initial conditions of the unmeasured state variables are frequently available. An identification criterion which is based on this a priori information is developed in the Appendix. The identification criterion can be written as:

$$
\boldsymbol{\Phi}\left(\hat{\boldsymbol{\theta}}, \hat{\boldsymbol{x}}_{0}, \hat{\boldsymbol{y}}_{1}, \ldots, \hat{\boldsymbol{y}}_{N}\right)=\frac{1}{2}[\hat{\boldsymbol{\theta}}-\boldsymbol{\theta}]^{T} \boldsymbol{\Omega}_{\boldsymbol{\theta}}^{-1}[\hat{\boldsymbol{\theta}}-\boldsymbol{\theta}]+\frac{1}{2} \sum_{i=1}^{N}\left[\boldsymbol{y}_{\boldsymbol{i}}-\hat{\boldsymbol{y}}_{\boldsymbol{i}}\right]^{T} \boldsymbol{\Omega}_{\boldsymbol{y}_{i}}^{-1}\left[\boldsymbol{y}_{\boldsymbol{i}}-\hat{\boldsymbol{y}}_{\boldsymbol{i}}\right]
$$

where $\boldsymbol{\Omega}_{\theta}$ is the a priori parameter covariance matrix, and $\boldsymbol{\Omega}_{\boldsymbol{y}_{i}}$ is the error covariance matrix (measurement and model equation errors).

The a priori parameter information is advantageous due to several reasons. First, initial parameter estimates are often available from off-line experiments or from estimates in previous time horizons. Secondly, the inclusion of a priori information in the identification criterion prevents the parameter estimation problem from becoming ill-conditioned, i.e. the problem becomes more robust with respect to missing process excitations and over-parameterization. Jang et al. (1987) use a least square criterion in the identification scheme, and they experience that the parameters become unidentifiable or nearly unidentifiable due to the lack of persistent process excitation.

The a priori parameter covariance matrix $\boldsymbol{\Omega}_{\theta}$ can either be computed from old data, or it may be treated as a tuning matrix (Ljungquist 1990). In the last case the parameter covariance matrix diagonal elements are set according to the amount of information in the measurements. If this information is significant due to natural excitations by disturbances or forced excitations, the corresponding diagonal elements of the covariance matrix are increased. This means that the confidence in the a priori parameter estimates are reduced compared to the confidence in the measurement information. 
The moving-horizon approach is used to reduce the optimization problem to one of a manageable dimension. The moving-horizon is defined from $t_{c}-N \Delta t$ to the current time $t_{c}$, where $\Delta t$ is the interval at which observations are available. The horizon length, $N \Delta t$, provides a means for tuning the performance of the identification scheme, but as a general rule it should be in the same range as the largest time constant of the system, i.e. to have enough dynamic information available for estimation.

\subsection{Orthogonal collocation on finite elements}

The optimization problem in the identification phase includes ordinary differential equations, and this problem can be converted to an algebraic optimization problem by discretizing the differential equations using orthogonal collocation on finite elements (Cuthrell and Biegler 1987, Villadsen and Michelsen 1978). The resulting algebraic equations can be written as equality constraints in a nonlinear programming formulation. This formulation allows process constraints to be emebedded directly. Lagrange interpolating polynomials are used in this paper to represent the continuous state profiles. Let $K$ represent the number of collocation points per finite element, then:

$$
\begin{gathered}
\hat{\boldsymbol{x}}_{K+1}^{i}(t)=\sum_{j=0}^{K} \hat{\boldsymbol{x}}_{[i j]} \boldsymbol{\Psi}_{[i j]}(t) \\
\boldsymbol{\Psi}_{[i j]}(t)=\prod_{k=0, k \neq j}^{K} \frac{\left(t-t_{[i k]}\right)}{\left(t_{[i j]}-t_{[i k]}\right)}=\prod_{k=0, k \neq j}^{K} \frac{\left(\tau-\tau_{k}\right)}{\left(\tau_{j}-\tau_{k}\right)} \\
t_{[i j]}=\alpha_{i}+\tau_{j}\left(\alpha_{i+1}-\alpha_{i}\right)=\alpha_{i}+\tau_{j} \Delta \alpha_{i}
\end{gathered}
$$

where $\hat{\boldsymbol{x}}_{K+1}^{i}(t)$ is a $(K+1)$ th order Lagrange polynomial at $i$ th finite element; $\hat{\boldsymbol{x}}_{[i j]}$ is the polynomial coefficient at $i$ th finite element and $j$ th collocation point $([i j] \equiv(i-1)(K+1)+j) ; \boldsymbol{\Psi}_{[i j]}(t)$ is a polynomial of degree $K$. Each finite element $\Delta \alpha_{i}$ is bounded by two knots, $\alpha_{i}$ and $\alpha_{i+1}$, and $\tau_{j}, \tau_{k}$ are the collocation points which are the shifted roots of the orthogonal Legendre polynomial of order $K$.

The residual equations at the collocation points are forced to satisfy the model equations exactly, and the polynomials between adjacent elements are forced to be continuous. These equations can be written as:

$$
\begin{gathered}
\boldsymbol{R}\left(\boldsymbol{t}_{[i j]}\right)=\sum_{k=0}^{K} \hat{\boldsymbol{x}}_{[i k]} \dot{\boldsymbol{\Psi}}_{k}\left(\tau_{j}\right)-\Delta \alpha_{i} \boldsymbol{f}\left(\hat{\boldsymbol{x}}_{[i j]}, \hat{\boldsymbol{u}}, \hat{\boldsymbol{v}}, \hat{\boldsymbol{\theta}}\right)=0 \quad i=1, \ldots, n_{\mathrm{el}} ; j=1, \ldots, K \\
\hat{\boldsymbol{x}}_{[10]}=\hat{\boldsymbol{x}}_{0} \quad \hat{\boldsymbol{x}}_{[10]}=\sum_{\mathrm{j}=0}^{K} \hat{\boldsymbol{x}}_{[l-1 j]} \boldsymbol{\Psi}_{j}(\tau=1) \quad l=2, \ldots, n_{\mathrm{el}}
\end{gathered}
$$

where $\dot{\boldsymbol{\Psi}}=d \boldsymbol{\Psi} / d \tau, \boldsymbol{\Psi}_{k}\left(\tau_{j}\right)=\Delta \alpha_{i} \boldsymbol{\Psi}_{[i k]}\left(t_{[i j]}\right)$, and $n_{\mathrm{el}}$ is the total number of finite elements. In this formulation the location of the knots can be based on the profile of the measured data; "steep" regions are approximated using more elements, while "flat" regions are approximated using less elements.

\section{Heat pump control example}

Heat pumps are used both in domestic and industrial applications, where heat is extracted from one substance and transferred to a second substance at a higher temperature. In domestic applications both the ambient temperature and the heat source temperature have a significant impact on the optimum performance of heat pumps. 


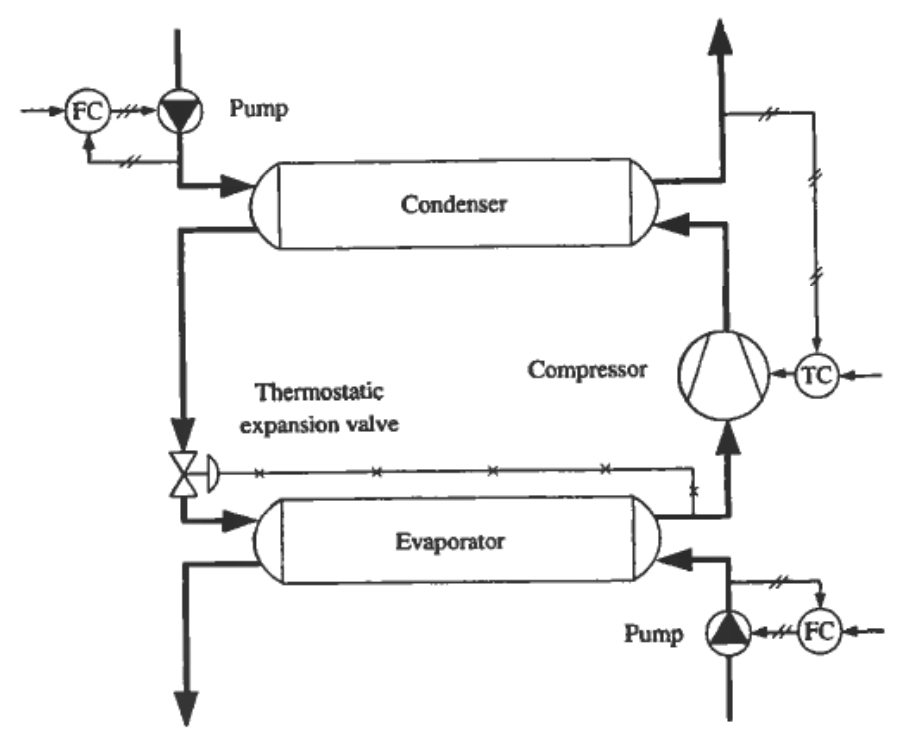

Figure 1. Flow chart of the heat pump unit.

These disturbances are relatively slow compared to the heat pump dynamics, and justify steady-state optimizing control. However, fast varying disturbances such as load variations, on-off control, discrete final control elements, oscillations, etc, are also present, and these will in some cases prevent the heat pumps from reaching any steady-state.

The proposed identification and optimization approach were evaluated using an experimental water-to-water heat pump unit at the Norwegian Institute of Technology. The flow chart of the heat pump unit is shown in Figure 1. The heat pump unit consists of a semi-hermetic variable-speed reciprocating compressor, a horizontal shell-andtube condenser including a subcooling unit, two thermostatic expansion valves, a dry expansion shell-and-tube evaporator, and two variable-speed centrifugal water pumps. The refrigerant is R-22. The nonlinear non-steady-state heat pump unit model which was used for process identification and control is given in Table 1. The steady-state part of the model is given in Table 2.

The heat pump unit regulatory control system consists of one temperature controller (TC), two flow controllers (FC), and two degree of superheat controllers (thermostatic expansion valves). The final control elements are the variable-speed compressor, the variable-speed water pumps, and the two expansion valves. The controlled variables are the condenser water outlet temperature, the water flow rates through the evaporator and condenser, and the degree of superheat in the suction line. The main objective of the regulatory control system is to maintain these controlled variables at their set-points.

The main objective of the heat pump unit optimizing control system is to find the optimum set-points for the temperature controller and the two flow controllers in the regulatory control system that minimize the total energy input (total electric power input) to the heat pump unit. The solution to this optimization problem must satisfy the heat pump unit load specification, and not violate the operational feasibility constraints. Table 3 summarizes the steady-state heat pump unit optimization problem. The 
Table 1. Heat pump unit non-steady-state model

$$
\begin{aligned}
& \text { Evaporator }\left(i=1, \ldots, n_{\mathrm{e}}\right) \text {, where } n_{\mathrm{c}}=3 \text { : } \\
& \frac{d T_{\mathrm{we}_{i}}}{d t}=\frac{k_{\mathrm{e}} A_{\mathrm{e}}}{\rho_{\mathrm{we}} V_{\mathrm{we}} c_{\mathrm{p}, \mathrm{we}}} \dot{m}_{\mathrm{r}}^{0.8}\left(T_{\mathrm{e}}^{\mathrm{sat}}-T_{\mathrm{we}_{i}}\right)+\frac{k_{\mathrm{se}} A_{\mathrm{se}}}{\rho_{\mathrm{we}_{\mathrm{e}}} V_{\mathrm{we}_{\mathrm{e}} c_{\mathrm{pe}}}} \dot{V}_{\mathrm{se}}^{0.6}\left[\frac{1}{2}\left(T_{\mathrm{se}_{i}-1}+T_{\mathrm{se}_{i}}\right)-T_{\mathrm{we}_{i}}\right] \\
& \frac{d T_{\mathrm{se}_{i}}}{d t}=\frac{k_{\mathrm{se}} A_{\mathrm{se}}}{\rho_{\mathrm{se}} V_{\mathrm{se}} c_{\mathrm{p}, \mathrm{se}}} \dot{V}_{\mathrm{se}^{0.6}}^{0 .}\left[T_{\mathrm{we}_{i}}-\frac{1}{2}\left(T_{\mathrm{se}_{i}-1}+T_{\mathrm{se}_{i}}\right)\right]+\frac{n_{\mathrm{c}}}{V_{\mathrm{se}}} \dot{\mathrm{se}}_{\mathrm{se}}\left(T_{\mathrm{se}_{i-1}}-T_{\left.\mathrm{se}_{i}\right)}\right. \\
& \frac{d p_{\mathrm{e}}}{d t}=\frac{k_{\mathrm{e}} A_{\mathrm{e}}\left(1 / n_{\mathrm{e}}\right) \dot{m}_{\mathrm{r}}^{0.8} \sum_{i}^{n_{\mathrm{e}}}{ }_{\mathrm{l}}\left(T_{\mathrm{we}_{i}}-T_{\mathrm{e}}^{\mathrm{sat}}\right)-\dot{m}_{\mathrm{r}}\left(h_{\mathrm{suc}}-h_{\mathrm{s}}\right)}{V_{\mathrm{ve}}\left(d \rho_{\mathrm{ve}}^{\mathrm{sat}} / d p_{\mathrm{e}}\right)\left(h_{\mathrm{ve}}^{\mathrm{sat}}-h_{\mathrm{le}}^{\mathrm{set}}\right)-V_{\mathrm{e}}}
\end{aligned}
$$

Condenser $\left(i=1, \ldots, n_{\mathrm{c}}\right)$, where $n_{\mathrm{c}}=2$ :

$$
\begin{aligned}
& \frac{d T_{\mathrm{wc}_{i}}}{d t}=\frac{k_{\mathrm{c}} A_{\mathrm{c}}}{\rho_{\mathrm{wc}} V_{\mathrm{wc}} c_{\mathrm{p}, \mathrm{wc}}} \dot{m}_{\mathrm{r}}^{-1 / 3}\left(T_{\mathrm{c}}^{\mathrm{sat}}-T_{\mathrm{wc}_{i}}\right)+\frac{k_{\mathrm{sc}} A_{\mathrm{sc}}}{\rho_{\mathrm{wc}} V_{\mathrm{wc}} c_{\mathrm{p}, \mathrm{wc}}} \dot{V}_{\mathrm{sc}}^{0.8}\left[\frac{1}{2}\left(T_{\mathrm{sc}_{i-1}}+T_{\mathrm{sc}_{i}}\right)-T_{\mathrm{wc}_{i}}\right] \\
& \frac{d T_{\mathrm{sc} i}}{d t}=\frac{k_{\mathrm{sc}} A_{\mathrm{sc}}}{\rho_{\mathrm{sc}} V_{\mathrm{sc}} c_{\mathrm{psc}}} \dot{V}_{\mathrm{sc}}^{0.8}\left[T_{\mathrm{wc}_{i}}-\frac{1}{2}\left(T_{\mathrm{sc}_{i}-1}+T_{\mathrm{sc}_{i}}\right)\right]+\frac{n_{\mathrm{c}}}{V_{\mathrm{sc}}} \dot{V}_{\mathrm{sc}}\left(T_{\mathrm{sc}_{i}-1}-T_{\left.\mathrm{sc}_{i}\right)}\right. \\
& \frac{d p_{\mathrm{c}}}{d t}=\frac{k_{\mathrm{c}} A_{\mathrm{c}}\left(1 / n_{\mathrm{c}}\right) \dot{m}_{\mathrm{r}}^{-1 / 3} \sum_{i}^{n_{\mathrm{c}}}{ }_{1}\left(T_{\mathrm{wc}_{i}}-T_{\mathrm{c}}^{\mathrm{sat}}\right)+\dot{m}_{\mathrm{r}}\left(h_{\mathrm{dis}}-h_{\mathrm{lc}}^{\mathrm{sat}}\right)}{V_{\mathrm{vc}}\left(d \rho_{\mathrm{vc}}^{\mathrm{sat}} / d p_{\mathrm{c}}\right)\left(h_{\mathrm{vc}}^{\mathrm{sat}}-h_{\mathrm{lc}}^{\mathrm{sat}}\right)-V_{\mathrm{c}}}
\end{aligned}
$$

Subcooler:

$$
\begin{gathered}
\frac{d T_{\mathrm{ws}}}{d t}=\frac{k_{\mathrm{s}} A_{\mathrm{s}}}{\rho_{\mathrm{ws}} V_{\mathrm{ws}} c_{\mathrm{p}, \mathrm{ws}}} \dot{m}_{\mathrm{r}}^{0.6}\left(T_{\mathrm{s}}-T_{\mathrm{ws}}\right)+\frac{k_{\mathrm{ss}} A_{\mathrm{ss}}}{\rho_{\mathrm{ws}} V_{\mathrm{ws}} c_{\mathrm{p}, \mathrm{ws}}} \dot{V}_{\mathrm{sc}}^{0-8}\left[\frac{1}{2}\left(T_{\mathrm{ss}, \mathrm{in}}+T_{\mathrm{ss}}\right)-T_{\mathrm{wss}}\right] \\
\frac{d T_{\mathrm{ss}}}{d t}=\frac{k_{\mathrm{ss}} A_{\mathrm{ss}}}{\rho_{\mathrm{ss}} V_{\mathrm{ss}} c_{\mathrm{p}, \mathrm{ss}}} \dot{V}_{\mathrm{sc}}^{0.8}\left[T_{\mathrm{ws}}-\frac{1}{2}\left(T_{\mathrm{ss}, \mathrm{in}}+T_{\mathrm{ss}}\right)\right]+\frac{1}{V_{\mathrm{ss}}} \dot{V}_{\mathrm{sc}}\left(T_{\mathrm{ss}, i n}-T_{\mathrm{ss}}\right) \\
\frac{d T_{\mathrm{s}}}{d t}=\frac{1}{\rho_{\mathrm{s}} V_{\mathrm{s}}} \dot{m}_{\mathrm{r}}\left(T_{\mathrm{c}}^{\mathrm{sat}}-T_{\mathrm{s}}\right)+\frac{k_{\mathrm{s}} A_{\mathrm{s}}}{\rho_{\mathrm{s}} V_{\mathrm{s}}\left(d h_{\mathrm{s}} / d T_{\mathrm{s}}\right)} \dot{m}_{\mathrm{r}}^{0.6}\left(T_{\mathrm{ws}}-T_{\mathrm{s}}\right)
\end{gathered}
$$

Thermostatic expansion valves: $\quad \Delta h_{\exp }=0 \quad T_{\text {suc }}-T_{\mathrm{e}}^{\mathrm{sat}}=$ const

Compressor: $\quad \dot{P}_{\mathrm{fc}}=\frac{\dot{m}_{\mathrm{r}}\left(h_{\mathrm{dis}}-h_{\mathrm{suc}}\right)}{\eta_{\mathrm{fc}}} \quad \eta_{\text {ise }}=\frac{h_{\text {ise }}-h_{\mathrm{suc}}}{h_{\mathrm{dis}}-h_{\mathrm{suc}}} \quad \dot{m}_{\mathrm{r}}=\frac{\dot{V}_{\mathrm{sw}} f_{\mathrm{fc}} \eta_{\mathrm{vol}}}{f_{\mathrm{ref}} v_{\mathrm{suc}}}$

Pumps: $\quad \dot{P}_{\mathrm{pe}}=\frac{\kappa_{\mathrm{se}}}{\eta_{\mathrm{pe}}} \dot{V}_{\mathrm{se}}^{3.0} \quad \dot{P}_{\mathrm{pc}}=\frac{\kappa_{\mathrm{sc}}}{\eta_{\mathrm{pc}}} \dot{V}_{\mathrm{sc}}^{3.0}$

operational feasibility constraints are given in Table 4, and are based on the following considerations:

- Protection against air leakage into the evaporator and compressor by keeping the low refrigerant pressure above atmospheric pressure. A lower limit on the suction pressure also prevents overheating of the hermetic motor.

- Protection against freezing of water inside the evaporator shell.

- Protection of the compressor against liquid entering from the evaporator through the suction line. This constraint is implicitly taken care of by the two degree of superheat controllers.

- Protection of the hermetic compressor motor and pump motors against overload.

- Protection against lubricant and refrigeration breakdown at the discharge side of the compressor. 
Table 2. Heat pump unit steady-state model

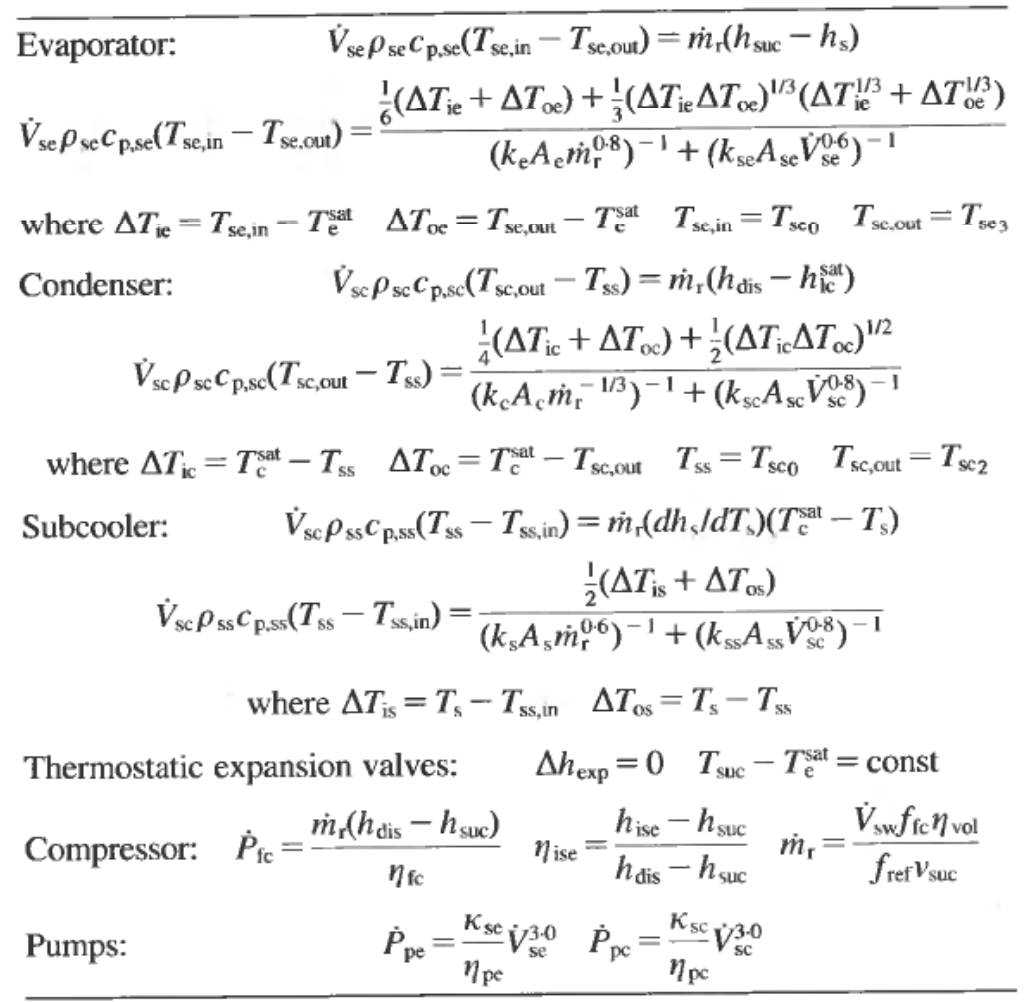

Table 3. Steady-state heat pump unit optimization problem

Given the disturbances $T_{\mathrm{se}, \text { in }}$ and $T_{\mathrm{ss}, \text { in }}$, and the load specification $\dot{E}_{\mathrm{hp}}$

Minimize $J=\dot{P}_{\mathrm{fc}}+\dot{P}_{\mathrm{pc}}+\dot{P}_{\mathrm{pc}}$

with respect to the freed variable vector $\left[\begin{array}{lll}T_{\mathrm{sc}, \text { out }} & \dot{V}_{\mathrm{se}} & \dot{V}_{\mathrm{sc}}\end{array}\right]^{T}$

subject to the equations in Table 2 with updated parameters and

$$
\dot{E}_{\mathrm{hp}}=\dot{V}_{\mathrm{sc}} \rho_{\mathrm{sc}} c_{\mathrm{p}, \mathrm{sc}}\left\{\left(T_{\mathrm{sc}, \text { out }}-T_{\mathrm{ss}, \text { in }}\right)-\mathscr{T}_{\mathrm{ref}} \ln \left[\mathscr{T}_{\mathrm{sc}, \text { out }} / \mathscr{T}_{\mathrm{ss}, \mathrm{in}}\right]\right\}
$$

and the inequality (operational feasibility) constraints

$$
\begin{aligned}
& g_{1}=1 \cdot 1-p_{\mathrm{c}} \quad g_{2}=\dot{P}_{\mathrm{pe}}-2 \cdot 2 \quad g_{3}=2 \cdot 0-T_{\mathrm{sc} . \mathrm{out}} \quad g_{4}=T_{\mathrm{dis}}-105 \cdot 0 \\
& g_{5}=\dot{P}_{\mathrm{fc}}-20 \cdot 5 \quad g_{6}=25 \cdot 0-f_{\mathrm{fc}} \quad g_{7}=f_{\mathrm{fc}}-75 \cdot 0 \quad g_{8}=p_{\mathrm{c}}-23 \cdot 0 \quad g_{9}=\dot{P}_{\mathrm{pc}}-2 \cdot 2
\end{aligned}
$$

- Speed reduction is limited $(25 \mathrm{~Hz})$ to prevent compressor lubrication, oil return, and motor overheating problems. The upper limit of compressor speed $(75 \mathrm{~Hz})$ is set according to degradation of compressor valves and bearings performance.

- High pressure protection of the condenser and compressor crankcase and housing.

\subsection{On-line identification}

The uncertain parameters of the steady-state heat pump unit model form the basis for the selection of parameters for on-line estimation. An important part of the 
Table 4. Operational feasibility constraints in the heat pump unit optimization

\begin{tabular}{|c|c|}
\hline \multicolumn{2}{|l|}{ Evaporator } \\
\hline Variable name & Constraint \\
\hline Evaporator water outlet temperature & Above $2 \cdot 0^{\circ} \mathrm{C}$ \\
\hline Evaporator pressure & Above $1 \cdot 1$ bar \\
\hline Pump motor electric power input & Below $2 \cdot 2 \mathrm{~kW}$ \\
\hline \multicolumn{2}{|l|}{ Compressor } \\
\hline Variable name & Constraint \\
\hline Compressor motor electric power input & Below $20 \cdot 5 \mathrm{~kW}$ \\
\hline Compressor motor frequency & $(25-75) \mathrm{Hz}$ \\
\hline Compressor discharge temperature & Below $105.0^{\circ} \mathrm{C}$ \\
\hline \multicolumn{2}{|l|}{ Condenser } \\
\hline Variable name & Constraint \\
\hline Condenser pressure & Below 23.0 bar \\
\hline Pump motor electric power input & Below $2 \cdot 2 \mathrm{~kW}$ \\
\hline
\end{tabular}

identification problem is to decide which of these parameters that are candidates for on-line adjustment. Table 5 shows the impact these parameters have on the optimum operation of the heat pump unit. Each of the parameters were individually perturbed and the model re-optimized for each perturbation. The inlet water temperature to the evaporator and condenser, the water flow rates through the heat exchangers, and the load specification were held constant, i.e. $T_{\mathrm{se}, \text { in }}=10 \cdot 0^{\circ} \mathrm{C}, T_{\mathrm{ss}, \mathrm{i}}=41 \cdot 1^{\circ} \mathrm{C}$, $\dot{V}_{\mathrm{se}}=1.8 \times 10^{-3} \mathrm{~m}^{3} / \mathrm{s}, \quad \dot{V}_{\mathrm{sc}}=1.4 \times 10^{-3} \mathrm{~m}^{3} / \mathrm{s}, \quad$ and $\quad \dot{E}_{\mathrm{hp}}=8.0 \mathrm{~kW}$. The thermal efficiency $\eta_{\mathrm{fc}}$ and the isentropic efficiency $\eta_{\text {ise }}$ of the compressor unit have the largest effect on the objective function. The thermal efficiency, the pump parameters, and the heat-transfer related parameters in the evaporator and condenser have the largest effect on the set-points. Several parameters have a significant effect on the operational feasibility constraints. However, this only becomes important in cases where these constraints are active or near active.

Two sets of parameters were estimated separately in the identification phase. The first set consisted of the degree of superheat in the suction line $\left(T_{\mathrm{suc}}-T_{\mathrm{e}}^{\mathrm{sat}}\right)$, and the pump model parameters $\left(\kappa_{\mathrm{se}} / \eta_{\mathrm{pe}}\right.$ and $\left.\kappa_{\mathrm{sc}} / \eta_{\mathrm{pc}}\right)$. These parameters were calculated from the mean values of the measurements in the most recent time horizon inserted in the respective model equations. The second set of parameters consisted of the heat-transfer related parameters in the evaporator $\left(k_{\mathrm{se}} A_{\mathrm{se}}\right.$ and $\left.k_{\mathrm{e}} A_{\mathrm{e}}\right)$, the evaporator shell volume $\left(V_{\mathrm{se}}\right)$, the water side heat-transfer related parameter in the condenser $\left(k_{\mathrm{sc}} A_{\mathrm{sc}}\right)$, the condenser water volume $\left(V_{\mathrm{sc}}\right)$, and the isentropic, thermal, and volumetric efficiency in the compressor unit.

The measurement vector $\boldsymbol{y}$ is divided into two parts:

$$
\boldsymbol{y}=\left[\begin{array}{l}
y^{\prime} \\
y^{\prime \prime}
\end{array}\right]
$$

where:

$$
\begin{aligned}
\boldsymbol{y}^{\prime} & =\left[\begin{array}{lllllll}
T_{\mathrm{se}, \text { out }} & p_{\mathrm{c}} & \dot{P}_{\mathrm{fc}} & T_{\mathrm{dis}} & T_{\mathrm{sc}, \text { out }} & p_{\mathrm{c}} & T_{\mathrm{s}}
\end{array}\right]^{T} \\
\boldsymbol{y}^{\prime \prime} & =\left[\begin{array}{lllllll}
T_{\mathrm{sc}, \text { in }} & \dot{V}_{\mathrm{se}} & \dot{P}_{\mathrm{pe}} & T_{\mathrm{suc}} & \dot{V}_{\mathrm{sc}} & T_{\mathrm{ss}, \text { in }} & \dot{P}_{\mathrm{pc}}
\end{array}\right]^{T}
\end{aligned}
$$

Only the first part of the measurement vector $\left(y^{\prime}\right)$ was reconciled, i.e. included in the 

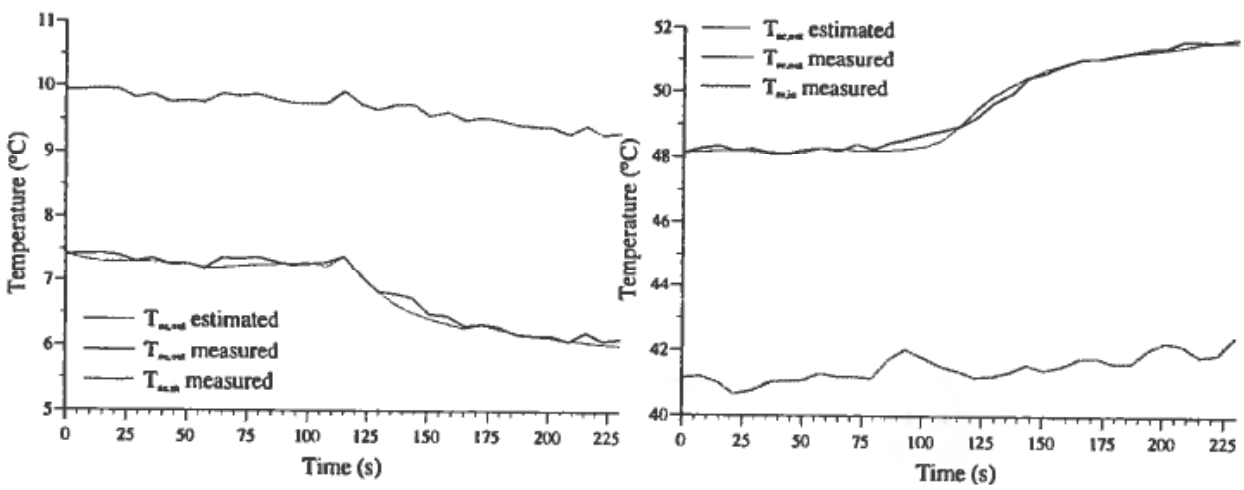

Figure 2. Evaporator and condenser outlet water temperature estimates to a step increase in compressor motor frequency.

identification criterion given in equation (6). The corresponding covariance matrix is given by:

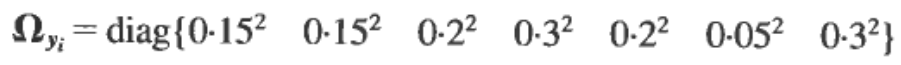

The second part of the measurement vector $\left(y^{\prime \prime}\right)$ was used as input variables to the identification scheme.

The identification algorithm was tested under different experimental conditions, and this case study shows the results from a step change in the compressor motor frequency. The time horizon was set to $57.6 \mathrm{~s}$ and the sampling time $\Delta t$ was $7.2 \mathrm{~s}(N=8)$. Seven interior collocation points $(K=7)$ and 1 finite element $\left(n_{\mathrm{el}}=1\right)$ were used for each time horizon. The time horizon movement was equal to $\mathrm{N}$ samples, i.e. no overlap between two subsequent time horizons. The initial conditions for the measured and unmeasured state variables were taken from measured values and previous time horizon estimates respectively.

Table 6 shows the parameters which were estimated on-line together with their a priori information. The parameters were assumed to be constant over each time horizon and the a priori parameter information was not updated from one time horizon to the next. Lower and upper bounds on the states and parameters were set according to
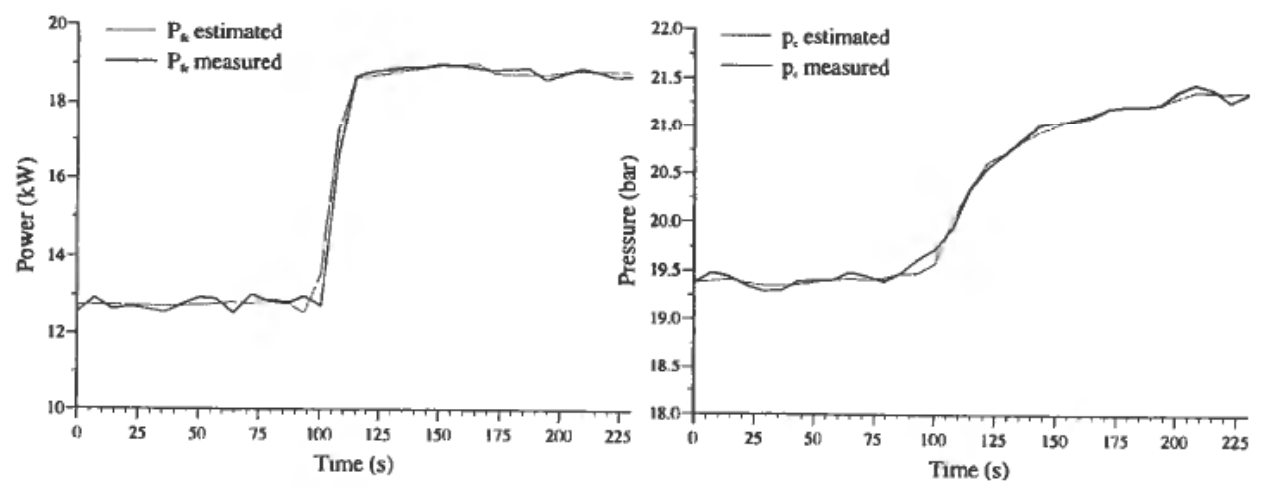

Figure 3. Power input to the compressor unit and condenser pressure estimates to a step increase in compressor motor frequency. 


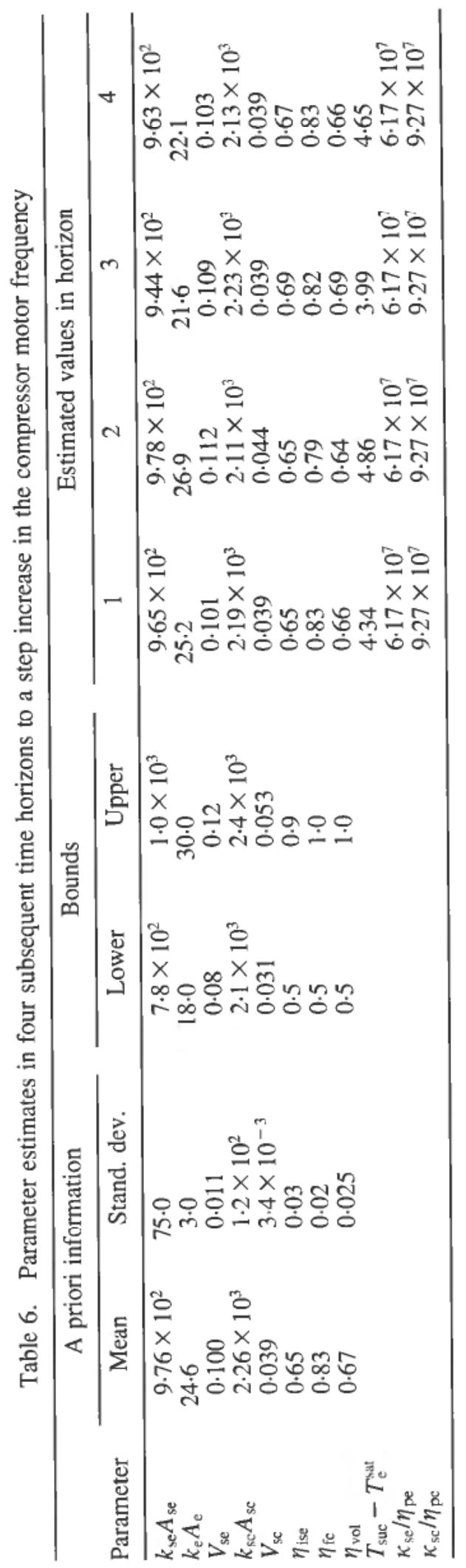




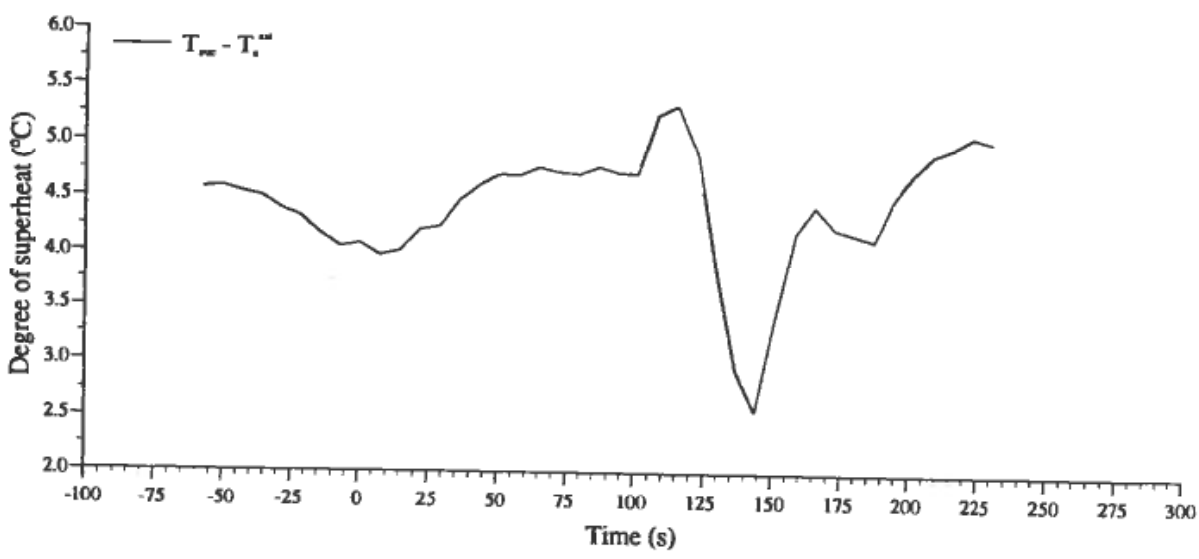

Figure 4. Measured degree of superheat in the suction line.

physical insight, and these bounds were easily implemented in the nonlinear programming approach.

Table 6 shows the estimated parameter values in 4 subsequent time horizons when the compressor motor frequency was stepped from 35 to $50 \mathrm{~Hz}$ at time $105 \mathrm{~s}$. The water flow rates through the evaporator and condenser were $\dot{V}_{\mathrm{se}}=3.00 \times 10^{-3} \mathrm{~m}^{3} / \mathrm{s}$ and $\dot{V}_{\mathrm{sc}}=1.45 \times 10^{-3} \mathrm{~m}^{3} / \mathrm{s}$ respectively. Figure 2 and Figure 3 compare the predicted and measured values of the evaporator and condenser outlet water temperatures, the compressor unit power input, and the condenser pressure. The condenser pressure, the condenser outlet water temperature and the power input to the compressor unit increased, while the evaporator outlet water temperature decreased when the compressor motor frequency was stepped from 35 to $50 \mathrm{~Hz}$. The reconciled data estimates in Figures 2 and 3 are in good agreement with the measured values, and verify the predictive capabilities of the heat pump unit model. The evaporator shell volume $\left(V_{\mathrm{se}}\right)$ and the condenser water volume $\left(V_{\mathrm{sc}}\right)$ were only identifiable when the evaporator and condenser were sufficiently excited. In the first and second time horizon only minor dynamic information was present, but the inclusion of the a priori parameter information in the identification criterion prevented the optimization problem from becoming ill-conditioned. In the third time horizon considerable transients were present, and this resulted in a slight over-estimation of the compressor unit isentropic and volumetric efficiency in this time horizon.

Some minor smearing effects were present, where modelling errors and to some extent measurement errors affected some of the state and parameter estimates. The degree of superheat controllers were unstable and oscillated as shown in Figure 4. The controller models were not included in the non-steady-state heat pump unit model and represented together with the assumed steady-state compressor unit model the dominant modelling errors. To reduce the smearing effects, the identification scheme must be further decentralized, where only those measurements which contain "information" about a parameter are used in the different identification criteria (Krishnan et al. 1992).

Optimization problems based on orthogonal collocation on finite elements are often sparse and lead to large-scale problems with few degrees of freedom. This case study contained 121 independent variables and 113 equality constraints. The SQP algorithm developed by Schittkowski (1985) was used to solve the optimization problem, and no attempt was made to utilize the sparsity of the problem. To increase the computational 


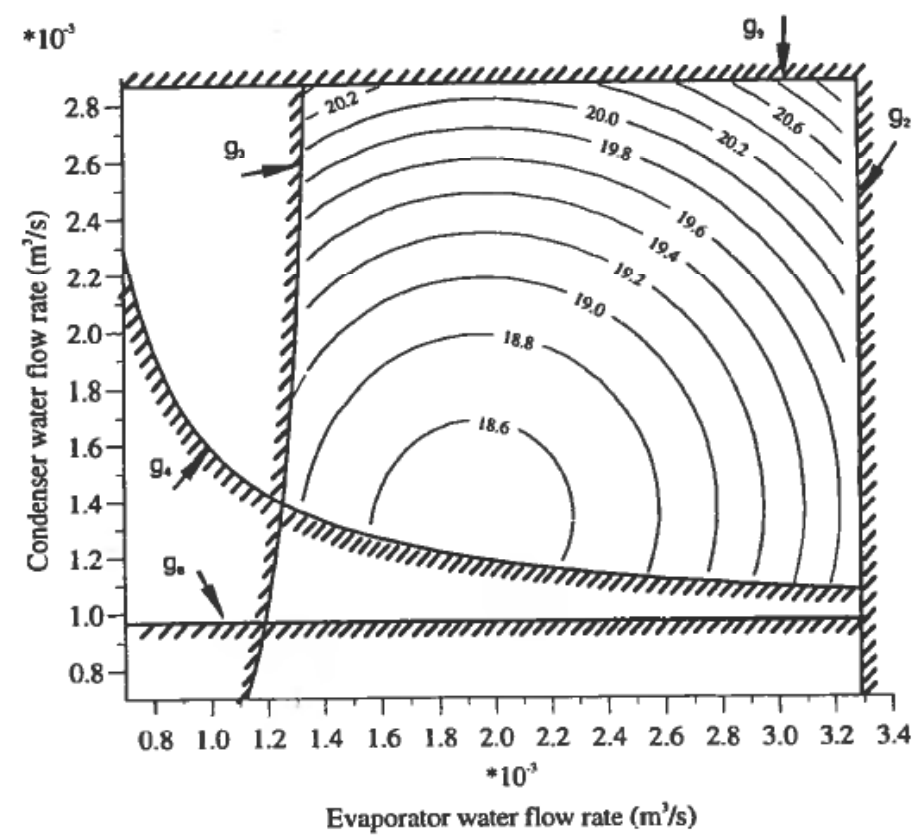

Figure 5. Contour and constraint diagram for the steady-state optimization problem.

efficiency and robustness of such large, sparse nonlinear programming problems, special purpose algorithms are needed. See Liebman et al. (1992) and Vasantharajan et al. (1988) for a discussion. The decentralization of the identification scheme discussed above will also reduce the dimension of the optimization problem, where smaller problems are solved sequentially.

Figure 5 shows the constraint and objective function contour diagram of the steady-state optimization problem (Table 3 ) with updated model parameters from the fourth time horizon. The evaporator and condenser water inlet temperatures were $9.5^{\circ} \mathrm{C}$ and $42.0^{\circ} \mathrm{C}$, and the heat pump unit load specification $\left(\dot{E}_{\mathrm{hp}}\right)$ was $8.0 \mathrm{~kW}$. The axis of the diagram are the water volume flow rates in the evaporator and condenser. Figure 5 shows that the feasible region of operation was constrained by the capacity limit of the pumps $\left(g_{2}\right.$ and $\left.g_{9}\right)$, the water freezing limit in the evaporator $\left(g_{3}\right)$, and the discharge temperature high limit $\left(g_{4}\right)$. The calculated optimum power input to the heat pump unit were $18.5 \mathrm{~kW}$ at $\dot{V}_{\mathrm{se}}=1.91 \times 10^{-3} \mathrm{~m}^{3} / \mathrm{s}$ and $\dot{V}_{\mathrm{sc}}=1.31 \times 10^{-3} \mathrm{~m}^{3} / \mathrm{s}$, and nooperational feasibility constraints were active at the optimum.

\section{Conclusions}

On-line steady-state optimizing control offers the prospect of improved economic performance for a wide range of processes. This paper has followed the approach of decomposing the identification and optimization phase, and has presented an algorithm for solving the general nonlinear dynamic identification problem. The robustness and the computational efficiency of the algorithm were increased by the inclusion of a priori parameter information in the identification criterion, and by the utilization of a simultaneous optimization and model solution strategy. Future research efforts will focus on increasing the efficiency of the current algorithm, and to define tuning and updating criteria for the a priori parameter information. 


\section{Appendix}

\section{A stochastic approach to state and parameter estimation}

The identification scheme developed below applies to state and parameter estimation in nonlinear ordinary vector differential-algebraic equations. By assuming an exact structural model, in which the true values (though unknown) of the state variable $\hat{\boldsymbol{x}}$, the measurements $\hat{\boldsymbol{y}}$, the parameters $\hat{\boldsymbol{\theta}}$, and the unmeasured initial conditions of the state variables $\hat{\boldsymbol{x}}_{0}$ apply exactly, we get:

$$
\begin{aligned}
\frac{d \hat{x}}{d t} & =f[\hat{x}(t), \hat{u}(t), \hat{v}(t), \hat{\boldsymbol{\theta}}] \\
\hat{\boldsymbol{x}}_{0} & =\hat{\boldsymbol{x}}\left(t_{0}\right) \\
\hat{\boldsymbol{y}}(t) & =z[\hat{\boldsymbol{x}}(t), \hat{\boldsymbol{u}}(t), \hat{\boldsymbol{v}}(t), \hat{\boldsymbol{\theta}}]
\end{aligned}
$$

The probability distribution function (pdf) of the measurement errors, $\boldsymbol{\varepsilon}_{y_{i}}=\boldsymbol{y}\left(t_{i}\right)-\hat{\boldsymbol{y}}\left(t_{i}\right)$, is given by $p_{i}\left(\boldsymbol{\varepsilon}_{y_{i}}\right)$. By assuming that the measurement errors in different samples are statistically independent the joint pdf for $N$ samples is given by:

$$
p\left(\boldsymbol{\varepsilon}_{\boldsymbol{y}_{1}}, \ldots, \boldsymbol{\varepsilon}_{\boldsymbol{y}_{N}}\right)=\prod_{i=1}^{N} p_{i}\left(\boldsymbol{\varepsilon}_{\boldsymbol{y}_{i}}\right)
$$

The assumption of an exact structural model is rarely true. This is due to neglecting effects in the model formulation which result in errors in the model equations. The dual nature of the model equation errors and measurement errors is ignored, and this implies that information about the model equation errors must be included in $p_{i}\left(\varepsilon_{y_{i}}\right)$. The likelihood function of $N$ samples becomes:

$$
L\left(\hat{y}_{1}, \ldots, \hat{y}_{N}\right)=\prod_{i=1}^{N} p_{i}\left(y_{i}-\hat{y}_{i}\right)
$$

where $\boldsymbol{y}_{i}=\boldsymbol{y}\left(t_{i}\right)$ is the measurement vector at time $t_{i}$, and $\hat{y}_{i}=\hat{y}\left(t_{i}\right)$ is constrained by the model equations.

The parameter vector is augmented to include the initial conditions of the unmeasured state variables, i.e. $\hat{\boldsymbol{\vartheta}}=\left[\hat{\boldsymbol{\theta}} \hat{\boldsymbol{x}}_{0}\right]^{T}$, and the prior distribution of $\hat{\boldsymbol{\vartheta}}$ is given by $p_{0}(\hat{\boldsymbol{\vartheta}})$. Bard (1974) use Bayes' theorem to find the posterior pdf:

$$
\mathrm{p}^{*}\left(\hat{\boldsymbol{\vartheta}}, \hat{\boldsymbol{y}}_{1}, \ldots, \hat{\boldsymbol{y}}_{N}\right)=c L\left(\hat{\boldsymbol{y}}_{1}, \ldots, \hat{\boldsymbol{y}}_{N}\right) p_{0}(\hat{\boldsymbol{\vartheta}})
$$

where $c$ is a constant. In general the probability density functions are unknown, and normal distribution functions are assumed. By assuming that errors in each sample are independent and distributed with the same known covariance matrix $\boldsymbol{\Omega}_{y_{i}}$ the pdf becomes:

$$
p_{i}\left(\boldsymbol{\varepsilon}_{\boldsymbol{y}_{i}}\right)=(2 \pi)^{-m / 2}\left|\boldsymbol{\Omega}_{y_{i}}\right|^{-1 / 2} \exp \left[-\frac{1}{2}\left(\boldsymbol{y}_{i}-\hat{\boldsymbol{y}}_{i}\right)^{T} \boldsymbol{\Omega}_{\boldsymbol{y}_{i}}^{-1}\left(y_{i}-\hat{\boldsymbol{y}}_{i}\right)\right]
$$

where $m=\operatorname{dim} \boldsymbol{y}_{i}$, and $\boldsymbol{\Omega}_{\mathbf{y}_{\mathrm{i}}}=\operatorname{diag}\left\{\sigma_{y_{n}}^{2} \ldots \sigma_{y_{i m}}^{2}\right\}$. By assuming that the augmented parameter vector is independent and distributed with known mean $\boldsymbol{\vartheta}$, and known covariance matrix $\boldsymbol{\Omega}_{\vartheta}$, the prior pdf becomes:

$$
p_{0}(\hat{\boldsymbol{\vartheta}})=(2 \pi)^{-p / 2}\left|\boldsymbol{\Omega}_{\vartheta}\right|^{-1 / 2} \exp \left[-\frac{1}{2}(\hat{\boldsymbol{\vartheta}}-\boldsymbol{\vartheta})^{T} \boldsymbol{\Omega}_{\vartheta}^{-1}(\hat{\boldsymbol{\vartheta}}-\boldsymbol{\vartheta})\right]
$$


where $p=\operatorname{dim} \boldsymbol{\vartheta}$, and $\boldsymbol{\Omega}_{\vartheta}=\operatorname{diag}\left\{\sigma_{\hat{\vartheta}_{1}}^{2} \ldots \sigma_{\vartheta_{p}}^{2}\right\}$. Maximizing the posterior pdf is equivalent to maximize its logarithm. The objective function to be minimized with all constants omitted becomes:

$$
\begin{aligned}
\boldsymbol{\Phi}\left(\hat{\boldsymbol{\theta}}, \hat{\boldsymbol{x}}_{0}, \hat{\boldsymbol{y}}_{1}, \ldots, \hat{\boldsymbol{y}}_{N}\right)= & \frac{1}{2}[\hat{\boldsymbol{\theta}}-\boldsymbol{\theta}]^{T} \boldsymbol{\Omega}_{\boldsymbol{\theta}}^{-1}[\hat{\boldsymbol{\theta}}-\boldsymbol{\theta}]+\frac{1}{2}\left[\hat{\boldsymbol{x}}_{0}-\boldsymbol{x}_{0}\right]^{T} \boldsymbol{\Omega}_{x_{0}}^{-1}\left[\hat{\boldsymbol{x}}_{0}-\boldsymbol{x}_{0}\right] \\
& +\frac{1}{2} \sum_{i=1}^{N}\left[\boldsymbol{y}_{\boldsymbol{i}}-\hat{\boldsymbol{y}}_{i}\right]^{T} \boldsymbol{\Omega}_{\boldsymbol{y}_{i}}^{-1}\left[\boldsymbol{y}_{\boldsymbol{i}}-\hat{\boldsymbol{y}}_{\boldsymbol{i}}\right]
\end{aligned}
$$

\section{REFERENCES}

[1] ARKun, Y. and Stephanopoulos, G. (1980). Optimizing Control of Industrial Chemical Processes: State of the Art Review. Proc. Joint Autom. Control. Conf., 1, WPS5-A.

[2] BAMBERGER, W. and ISERMANN, R. (1978). Adaptive On-Line Steady State Optimization of Slow Dynamic Processes. Automatica, 14.

[3] BARD, Y. (1974). Nonlinear Parameter Estimation, Academic Press.

[4] BIEGLER, L. T. and RAWLINGS, J. B. (1991). Optimization Approaches to Nonlinear Model Predictive Control. In Y. Arkun and W. H. Ray, eds, Proceedings of the Fourth International Conference on Chemical Process Control, Padre Island, Texas, February $17-22$.

[5] BOX, G. E. P. and DRAPER, N. R. (1968). Evolutionary Operation, John Wiley.

[6] BRIT, H. I. and LUECKE, R. H. (1973). The Estimation of Parameters in Nonlinear, Implicit Models. Technometrics, 15.

[7] Cuthrell, J. E. and BiEGLER, L. T. (1987). On the Optimization of Differential-Algebraic Process Systems. AIChE Journal, 33.

[8] EATON, J. W. and RAWLings, J. B. (1990). Feedback Control of Chemical Processes Using On-Line Optimization Techniques. Computers Chem Engng., 14.

[9] Edgar, T. F. and HimmelblaU, D. M. (1989). Optimization of Chemical Processes, McGraw-Hill.

[10] EDLER, J., NiKIFoRUK, P. N. and TinKER, E. B. (1970). A Comparison of the Performance of Techniques for Direct, On-Line Optimization. The Canadian J. of Chem. Eng., 48.

[11] JANG, S.-S., JoSEPH, B. and MUKaI, H. (1987). On-Line Optimization of Constrained Multivariable Chemical Processes. AIChE Journal, 33.

[12] JANG, S.-S., JOSEPH, B. and MUKAI, H. (1986). Comparison of Two Approaches to On-Line Parameter and State Estimation of Nonlinear Systems. Ind. Eng. Chem. Process Des. Dev., 25.

[13] Kim, I.-W., LIEBMAN, M. J. and EdGAR, T. F. (1990). Robust Error-in-Variables Estimation Using Nonlinear Programming Techniques. AIChE Journal, 36.

[14] KIM, I.-W., Liebman, M. J. and EdGaR, T. F. (1991). A Sequential Error-in-Variables Method for Nonlinear Dynamic Systems. Computers Chem. Engng., 15.

[15] Krishnan, S., Barton, G. W. and Perkins, J. D. (1992). Robust Parameter Estimation in On-Line Optimization-Part I. Methodology and Simulated Case Study. Computers Chem. Engng., 16.

[16] LieBMAN, M. J., Edgar, T. F. and Lasdon, L. S. (1992). Efficient Data Reconciliation and Estimation for Dynamic Processes Using Nonlinear Programming Techniques. Computers Chem. Engng., 16.

[17] LJUNGQUIST, D. (1990). Online Estimation in Nonlinear State-Space Models with Application to Catalytic Cracking, Dr. ing. Thesis, The Norwegian Institute of Technology.

[18] MACDONALD, R. J. and HowAT, C. S. (1988). Data Reconciliation and Parameter Estimation in Plant Performance Analysis. AIChE Journal, 34.

[19] Narasimhan, S., MaH, R. S. H., Tamhane, A.C., Woodward, J. W. and Hale, J. C. (1986). A Composite Statistical Test for Detecting Changes of Steady States. AIChE Journal, 32.

[20] NARASIMHAS, S. and MAH, R. S. H. (1987). Generalized Likelihood Ratio Method for Gross Error Identification. AIChE Journal, 33.

[21] SCHITTKOWSKI, K. (1985). NLPQL: A Fortran Subroutine Solving Constrained Nonlinear Programming Problems. Annals of Operations Research, 5.

[22] Sistu, P. B. and BequetTe, B. W. (1990). Process Identification using Nonlinear Programming Techniques. Proc. Amer. Control Conf. 
[23] Sistu, P. B. and Bequette, B. W. (1991). Nonlinear Predictive Control of Uncertain Processes: Application to a CSTR. AIChE Journal, 37.

[24] VilladSEN, J. and Michelsen, M. L. (1978). Solution of Differential Equation Models by Polynomial Approximation, Prentice-Hall.

[25] Vasantharajan, S. and BIEGLER, L. T. (1988). Large-Scale Decomposition for Successive Quadratic Programming. Computers Chem. Engng., 12.

\section{Nomenclature}

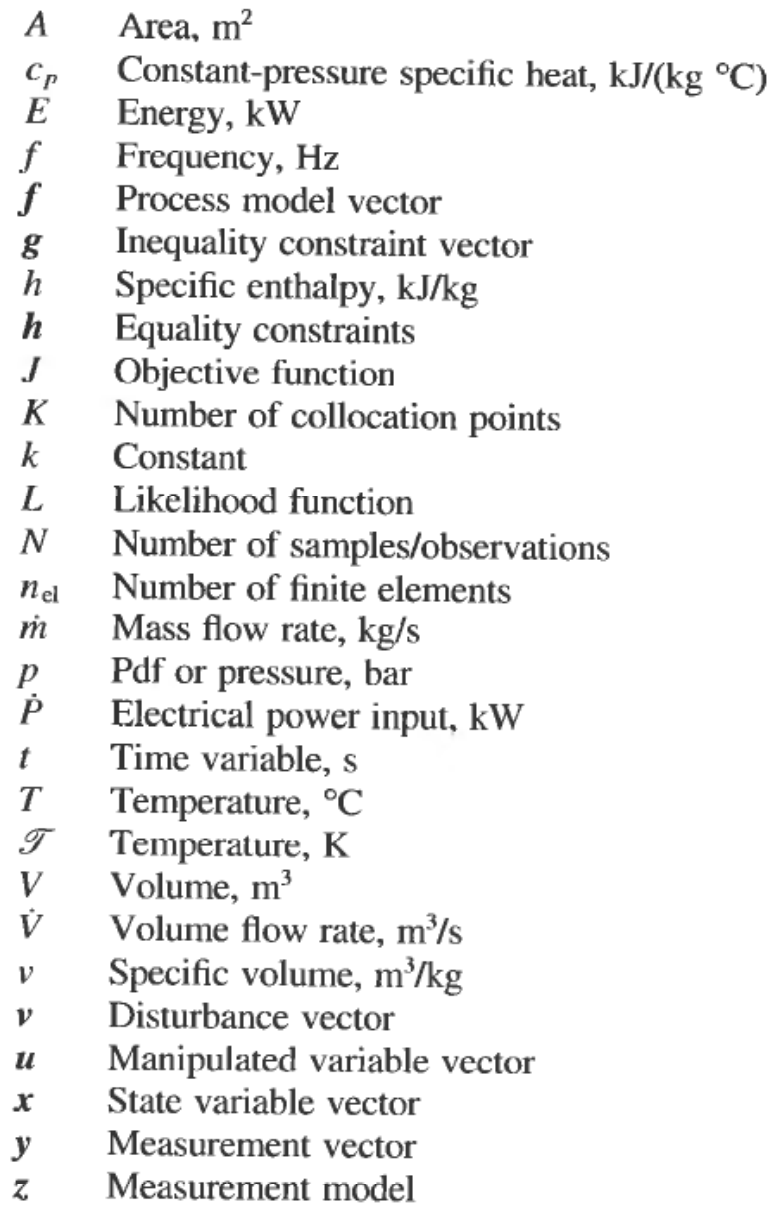

Greek Letters

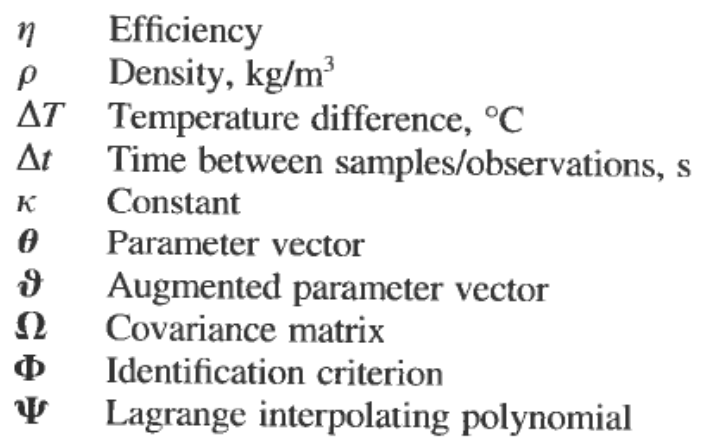




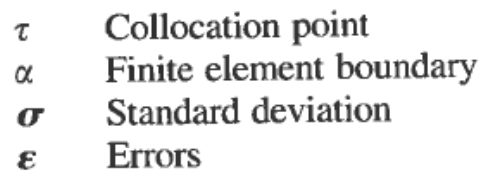

\section{Subscripts}

c Condenser

dis Discharge

e Evaporator

el Finite element

exp Expansion

fc Frequency converter and compressor

hp Heat pump

ic Inlet condenser

ie Inlet evaporator

in Inlet

is Inlet subcooler

ise Isentropic

Ic Liquid condenser

le Liquid evaporator

liq Liquid

oc Outlet condenser

oe Outlet evaporator

os Outlet subcooler

out Outlet

pc Pump condenser

pe Pump evaporator

$r$ Refrigerant

ref Reference

s Subcooled

sc Secondary condenser

se Secondary evaporator

suc Suction

ss Secondary subcooler

sw Swept

vc Vapour condenser

ve Vapour evaporator

vol Volumetric

wc Wall condenser

we Wall evaporator

ws Wall subcooler

$0 \quad$ Initial time or prior

Superscripts

sat Saturation

astimate

* Posterior 